\title{
Science performance of the Pupil-mapping Exoplanet Coronagraphic Observer (PECO)
}

\author{
Kerri Cahoy*a, Olivier Guyon ${ }^{\mathrm{b}}$, Glenn Schneider ${ }^{\mathrm{b}}$, Mark Marley ${ }^{\mathrm{a}}$, Ruslan Belikov ${ }^{\mathrm{a},}$ Michael Meyer ${ }^{\mathrm{b}}$, \\ Stephen Ridgway ${ }^{\mathrm{c}}$, Wesley Traub ${ }^{\mathrm{d}}$, Neville Woolf ${ }^{\mathrm{b}}$ \\ ${ }^{a}$ NASA Ames Research Center, Moffett Field, CA; ${ }^{b}$ University of Arizona, Tucson, AZ; ${ }^{c}$ National \\ Optical Astronomy Observatory, Tucson, AZ; ${ }^{\mathrm{d} J e t}$ Propulsion Laboratory, Pasadena, CA.
}

\begin{abstract}
The Pupil-mapping Exoplanet Coronagraphic Observer (PECO) medium-class mission concept is a 1.4-m space-based optical telescope with a high-performance Phase-Induced Amplitude Apodization (PIAA) coronagraph. PECO detects and characterizes exoplanets and their host systems at $2 \lambda / \mathrm{D}\left(0.15^{\prime \prime}\right)$ separation at high contrast ( 1e-10). The optical design images in 16 filter bands from 400-800 nm, producing simultaneous low-resolution target spectra. PECO will characterize terrestrial planets in the habitable zones of $\sim 20$ nearby F, G, K stars at spectral resolution of R 15, as well as over a dozen radial-velocity planets and over a hundred gas giants and exozodiacal dust disks. We discuss PECO's expected science performance and simulated data products over its three-year mission lifetime.
\end{abstract}

Keywords: PIAA, exoplanet, coronagraph, telescope, apodization, optical astronomy, PECO, ASMCS

\section{INTRODUCTION}

This paper describes the motivation behind the Pupil-Mapping Exoplanet Coronagraphic Observer (PECO) mission for direct imaging of exoplanets and their host systems and uses results from a detailed software simulation of the PIAA coronagraph to evaluate PECO's expected science performance over a three-year mission lifetime [1].

\subsection{PECO's role in exoplanet exploration}

PECO's space-based telescope uses a high-performance coronagraph to efficiently block the parent star's light and directly image a diverse population of exoplanets and their host systems, with an emphasis on having sufficient sensitivity to detect and characterize Earth-like planets within the habitable zones of over a dozen nearby F, G, K stars (the Sun is a G2V star). Optical images of terrestrial exoplanets are important for evaluating habitability because they provide measurements of the albedo and absorption spectra from starlight reflected by the exoplanet. The albedo and absorption features in these spectra yield information about the planet's atmospheric composition and thermal structure. For example, features in the optical for Earth include ozone (O3), molecular oxygen $(\mathrm{O} 2)$, and water $(\mathrm{H} 2 \mathrm{O})[2,3]$. Palle et al. (2009) show that even at coarse resolution, Rayleigh scattering, $\mathrm{O} 2$ and $\mathrm{H} 2 \mathrm{O}$ features appear in Earth's reflected spectra [3]. Figure 1 shows that the Solar System giant planets are distinguishable using R 15 albedo data from [4].
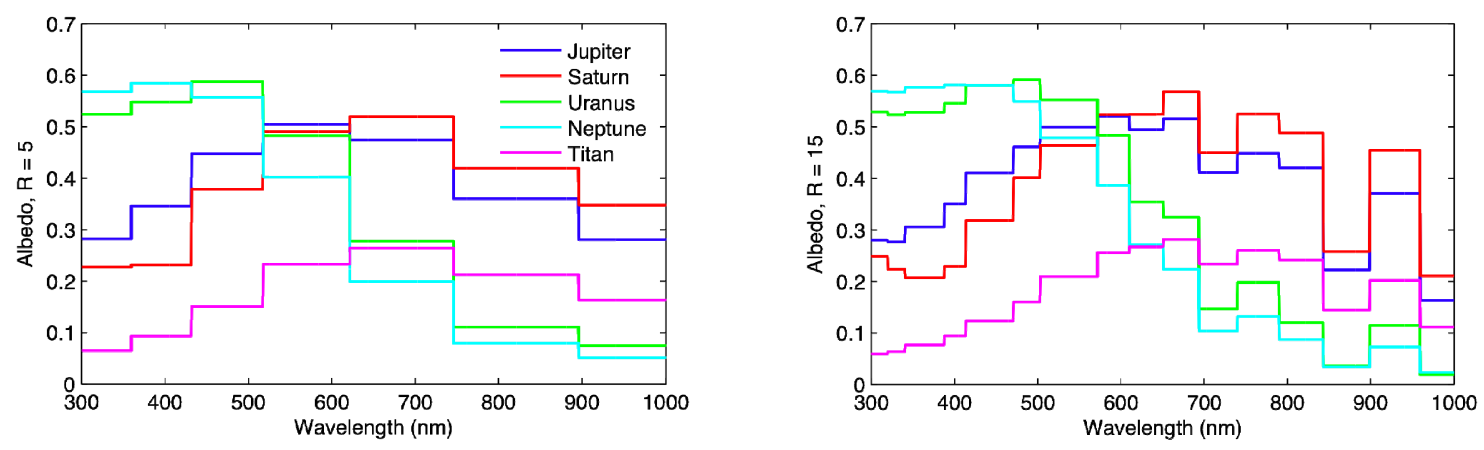

Figure 1. Albedo spectra from [4] at $\mathrm{R}=\lambda / \Delta \lambda=5$ (left) and $\mathrm{R}=15$ (right). For both, the Solar System gas giants (Jupiter, Saturn) are clearly distinguishable from the "ice" giants (Uranus, Neptune) and from Titan, a moon of Saturn with a dense nitrogen and methane atmosphere [5]. All planets are darker than Rayleigh (0.75) or Lambert (0.66) spheres.

Techniques and Instrumentation for Detection of Exoplanets IV, edited by Stuart B. Shaklan, Proc. of SPIE Vol. 7440, 74400G · C 2009 SPIE · CCC code: 0277-786X/09/\$18 · doi: 10.1117/12.826608 
Direct imaging of exoplanets using a space-based coronagraph complements and extends other methods of exoplanet detection and characterization. For example, a medium-class space-based coronagraph such as PECO will be able to perform follow-up characterizations of several nearby systems with planets already detected by the radial velocity method, such as 55 Cancri [6], as well as follow up on existing and future detections made by photometric missions such as Kepler and CoRoT, and help constrain the exoplanet radii, orbits, compositions, and temperatures. PECO's Earthtrailing heliocentric orbit permits high contrast observations without atmospheric correction and with longer duration single-instrument integrations to build up the signal to noise ratio (SNR) than diurnal cycle limited ground-based observations. These benefits trade against the size, mass, and cost limitations of spacecraft launch vehicles. Although smaller than current and planned optical and near-infrared space telescopes, PECO's design is optimized for the detection and characterization of exoplanets, including terrestrial planets within the habitable zones of nearby stars. PECO's PIAA coronagraph has higher contrast and coronagraphic throughput and a smaller inner working angle (IWA) [7] than coronagraphs on other current or planned optical/near-infrared space telescopes (e.g., Hubble Space Telescope $[8,9,10]$, James Webb Space Telescope [11]).

\subsection{Terminology}

Planet-star angular separations are discussed in terms of $\lambda / D$, the ratio of wavelength to primary diameter, as well as in terms of milliarcseconds (mas). The coronagraph's inner working angle (IWA) refers to the smallest planet-star separation possible at the contrast level desired for detection and characterization of the planet at the observed wavelength. Table 1 can be used as a quick reference for how IWA scales in terms of both $\lambda / \mathrm{D}$ and milliarcseconds as a function of $\lambda$ for $\mathrm{D}=1.4 \mathrm{~m}$. The outer working angle (OWA) refers to the largest separation between the center and edge of the image. In this work, we consider PECO's science performance for an IWA of $2 \lambda / \mathrm{D}$. The simulations use an OWA of $20 \lambda / \mathrm{D}$, however, implementation of the PECO's design is expected to have OWA $\sim 24 \lambda / \mathrm{D}$; the instrument OWA currently depends on the dimensions and number of actuators on the deformable mirrors (DMs) used for wavefront control [1].

In this work, usage of the term "Earths" refers to $1 \mathrm{RE}$ planets at a separation equivalent to $1 \mathrm{AU}$ from their parent stars with albedo 0.2, and "Super-Earths" refers to 2 RE planets at a separation of 1 AU with albedo 0.2. "Jupiters" refers to 1 RJ planets at a separation of $5 \mathrm{AU}$ with albedo 0.5. Planet-star separations are scaled to their Solar System equivalents based on the parent star's luminosity (see Table 3). The term "habitable zone" (HZ) in this work refers to the equivalent of $1 \mathrm{AU}$ separation for the target system being discussed, unless noted otherwise.

In addition to planets, target star systems also have background contributions in the optical from their dust and debris disks, as does our own Solar System. The PECO simulations take the contribution of our local zodiacal dust into account as a function of ecliptic latitude and also assume the target systems have their own zodiacal dust distributions, exozodi, that are scaled with respect to that in our Solar System, 1 zodi with $\mathrm{m}_{\mathrm{V}} \sim 22 \operatorname{arcsec}^{-2}[12,16]$.

\subsection{Contrast rationale}

The contrast required to image an exoplanet depends on the physical properties and orbital dynamics of the system, the instrumentation, and the background. A simple way of estimating the required contrast is to assume that the planet reflects its incident starlight equally in all directions, like a Lambertian sphere. As a function of planetary phase angle $\alpha$, the contrast can be expressed as [13]:

$$
C(\alpha)=\frac{2}{3} A\left(\frac{r}{a}\right)^{2}\left[\frac{\sin \alpha+(\pi-\alpha) \cos \alpha}{\pi}\right]
$$

Where $A$ is the planet's Bond albedo, $r$ radius, and $a$ orbital distance. For an Earth-like planet at quadrature $(\alpha=\pi / 2)$ orbiting a Sun-like star, $A \sim 0.3, r=6371 \mathrm{~km}$, and $a=1.5 \mathrm{e} 8 \mathrm{~km}$, so the contrast ratio is $\sim 1.15 \mathrm{e}-10$. In general, planetary atmospheres are not perfect scatterers, orbital distances and inclinations vary, and as noted for Figure 1, albedo is a function of wavelength and depends on the composition and structure of the planet's atmosphere. 


\subsection{Overview of the PECO spacecraft and optical design}

An Astrophysics Strategic Concept Mission Study (ASCMS), PECO is a medium-class 1.4$\mathrm{m}$ diameter space-based coronagraphic telescope in the same type of stable heliocentric Earth-trailing orbit as that used by Spitzer and Kepler. The PIAA coronagraph achieves 1e10 contrast at the second Airy ring $(0.15$ " at $550 \mathrm{~nm})$ by remapping the telescope pupil, as described in detail in $[13,14,15]$. The pupil remapping uses nearly all of the light coming into the aperture to achieve the full diffraction-limited resolution of the unvignetted aperture. The PIAA coronagraph has a theoretical throughput of $\sim 90 \%$ excluding losses due to optical coatings [16].

The design of PECO's science instrument takes full advantage of the high throughput and small IWA by simultaneously collecting all photons from 400 to $900 \mathrm{~nm}$ in 4 parallel coronagraph channels each with 4 sub-bands for a total of 16 science bands. After emerging from the beam compressor optics following the secondary mirror, dichroics split the light into four nearly identical channels, differing only in the physical size of the focal plane mask, the plate scale at the science detector, and the prescription of the pupil reimaging hyperbolas necessary to locate the pupil on one of the two deformable mirrors (DMs) which also serves as a fine-guidance mirror. A simplified diagram of the optical design is shown in Figure 2 and discussed in more detail in [1].

The wavefront control subsystem in each of the four spectral channels consists of two DMs and a coronagraphic low order wavefront sensor (CLOWFS) for accurate measurement of pointing errors and low order aberrations [17]. The two DMs per channel provide the degrees of freedom needed to correct both phase and amplitude errors such that primary PIAA mirror manufacturing requirements are consistent with those already proven for existing space telescope optics [18]. A set of two aspheric mirrors and a conventional pupil apodizer are used in each channel to fully apodize the telescope beam. The DMs are placed upstream of the PIAA optics, allowing them to correct a "dark hole" high-contrast region out to a $\sim 20 \lambda / \mathrm{D}$ OWA, about $1.6 \mathrm{arcsec}$ in the visible (see Table 1). An off-axis parabola focuses the apodized beam onto a coronagraph mask that blocks the central beam and reflects an annular beam extending to $\sim 2 \lambda / \mathrm{D}$ to the LOWFS camera. A simpler, lowerquality inverse PIAA system reverses the coma-like aberration in the beam to form a sharp image of the planet across the field [14, 19, 20]. Before reaching the detector, the beam is split into two linear polarizations by a Wollaston prism and four spectral channels with dichroics. There are thirty-two separate images formed on the detectors in all.

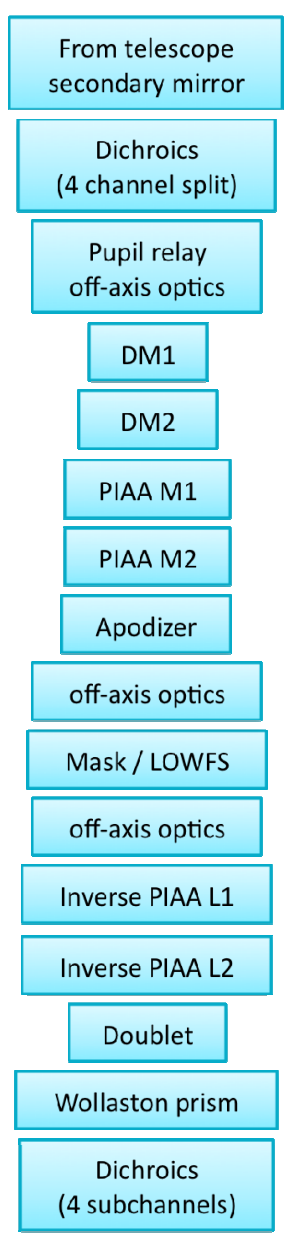

Figure 2: Simplified PECO optical layout

As discussed in more detail in Section 2, the three science requirements that drive PECO's instrument contrast, contrast stability, and spectral calibration requirements are: (i) detect an Earth-like planet having a contrast of 1e-10 with SNR > 4, (ii) measure spectral features of a Super-Earth that are $10 \%$ deep with SNR $>3$, and (iii) measure relative flux at three widely separated wavelengths to $3 \%$. The absolute calibration of scattered light in the dark hole to a level of $2.5 \mathrm{e}-11$ is driven by (i); the integration time becomes prohibitive when the background is much brighter than the planet, requiring the instrument scatter level to be kept to 1e-10. Relative spectral calibration to $\mathrm{SNR}=30$ is required by both (ii) and (iii), which translates to a relative calibration across the bandpass of 1.3e-11 for Super-Earths having a contrast of 4e-10. The flow-down of these requirements to the instrument is based on the detailed modeling in [21].

\section{SCIENCE GOALS}

PECO focuses on the characterization of exoplanets and their host systems. While this does include the goal of directly imaging and characterizing an Earth-like planet within the habitable zone of a nearby Sun-like star, PECO also addresses the important task of characterizing gas giants and radial-velocity targets as well as the debris disks and exozodiacal dust distributions of systems within its reach. The science goals lead to the detailed measurement and coronagraph requirements for PECO, which are described in more detail in $[1,22]$. In this section, we briefly describe PECO's science goals, and in Section 3, we discuss the performance simulations that align these science goals with mission capabilities and resources. Section 4 discusses an initial design reference mission for PECO that combines the mission design framework and science goals with the results from the performance simulations. 


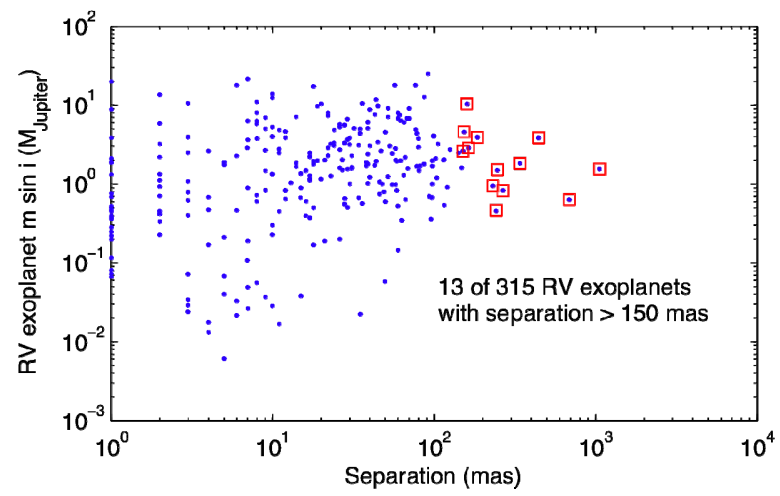

Figure 3: Separation (mas) vs. Msini of radialvelocity exoplanets [23]. Red squares show separations $>150$ mas.

\subsection{Terrestrial planets (Earths and Super-Earths)}

One of PECO's primary goals is to be able to detect and characterize Earth-like planets within the habitable zones of nearby Sun-like stars. As discussed in Section 1.3, this would require a contrast ratio $\sim 1 \mathrm{e}-10$, with $\mathrm{SNR}>5$ satisfying the criteria for detection. At a distance of $10 \mathrm{pc}$, a Sun-like system with an Earth-like planet at 1 AU would have a separation of 100 mas. Table 1 shows that with $\mathrm{D}=$ $1.4 \mathrm{~m}$, PECO's terrestrial candidate systems will likely be at distances $<10 \mathrm{pc}$, although the luminosity of the target star may increase this range (some cases may have a larger $\mathrm{HZ}$ separation). PECO's science goal is to detect Earths or Super-Earths with SNR $>4$ at the equivalent of $1-1.8$ AU from their parent stars at distances of 1.35 to $6 \mathrm{pc}$.

PECO's characterization goals are targeted toward a Solar System analog at $5 \mathrm{pc}$. More distant systems with larger terrestrial planets or larger HZs due to higher stellar luminosity will be detectable with a system designed for this $5 \mathrm{pc}$ Solar-System analog scenario.

To characterize Earths and Super-Earths and to evaluate habitability, PECO has a goal of detecting H2O, O2, or O3 in their atmospheres. The primary bands in the visible are water vapor near $720+/-20 \mathrm{~nm}$ and $825+/-20 \mathrm{~nm}$, oxygen at $760+/-10 \mathrm{~nm}$, and the ozone Chappuis band at $590+/-50 \mathrm{~nm}$. The ozone features in the Earth reflection spectra in [3] appear to occur in a region that also shows the effect of Rayleigh scattering. Detection of these absorption features is defined as the ability to measure the continuum with $\mathrm{SNR}=30$ and measure a $10 \%$ deep absorption feature at $\mathrm{R} \sim 10-20$ at $\mathrm{SNR}=3$.

Measurements of the continuum at multiple spectral intervals are important to characterize the albedo and colors of terrestrial planets. In conjunction with the habitability goal, PECO has a goal of measuring the continuum at the three spectral intervals; targeting the intervals between the $\mathrm{H} 2 \mathrm{O}, \mathrm{O} 2$, and $\mathrm{O} 3$ absorption features would be particularly useful.

PECO also has science goals to measure the brightness of a terrestrial planet at $10 \mathrm{pc}$ in at least one bandpass to within $25 \%$ (more likely to happen at shorter wavelengths) and to constrain the positions and orbits of detected terrestrial planets within the HZs of their parent stars. In addition to making multiple brightness measurements of the detected planet, the position goal is to constrain the planet's position to within about 30 mas (equivalent to $0.3 \mathrm{AU}$ for a system at $10 \mathrm{pc})$.

\subsection{Radial Velocity Follow-up}

The majority of the more than 300 exoplanets detected with the radial velocity (RV) method to date [23] are at relatively small angular separations from their parent stars from the perspective of a medium-class coronagraphy mission. Figure 3 shows the baker's dozen of RV exoplanets with separations greater than 150 mas. As radial-velocity methods become more sensitive, detections of lower-mass planets at larger separations will increase.

A direct imaging mission provides valuable follow-up on these RV exoplanets, without spending additional mission time in the search and detect phase. Multiple direct imaging observations that are well-separated will constrain both orbits and masses by providing the inclination such that the masses can be decoupled from the radial velocity Msin $i$ measurement. As RV technology continues to develop, using PECO to directly image RV exoplanets aligns well with the goal of directly imaging terrestrial exoplanets in the HZ of their parent star (e.g., as HARPS upgrades, and laser comb calibration comes online for RV measurements [24]).

Data in Figure 3 show that the Msini of the RV exoplanets that PECO can detect are on the order of the mass of Jupiter. Their characterization would provide a useful benchmark for the detection and characterization of other extrasolar giant planets (EGPs). PECO therefore has a science goal of characterizing the spectral features of at least 5 of the known RV extrasolar giant planets. Section 2.3 discusses the details of this requirement in context with the other EGP science goals. 
Table 2: Summary of PECO science goals as described in Section 2.

\begin{tabular}{|c|c|c|}
\hline Terrestrial planets & Extrasolar Giant Planets & Debris Disks and Exozodiacal Dust \\
\hline $\begin{array}{l}\text { At } 1.35-6 \text { pc, detect }(\mathrm{SNR}>5) \text { and } \\
\text { characterize }(\mathrm{SNR}>30) \text { Earths and Super- } \\
\text { Earths in the HZs of } \mathrm{F}, \mathrm{G}, \mathrm{K} \text { stars. } \\
\text { At } 5 \mathrm{pc} \text {, detect } \mathrm{H} 2 \mathrm{O}, \mathrm{O} 2 \text {, or } \mathrm{O} 3 \text { for a Super- } \\
\text { Earth in the } \mathrm{HZ} \text { of an } \mathrm{F}, \mathrm{G}, \mathrm{K} \text { star. } \\
\text { Measure continuum with SNR }>30 \\
\text { and a } 10 \% \text { deep absorption feature } \\
\text { with SNR }=3 \text { and } \mathrm{R} \sim 10-20 \text {. } \\
\text { At } 10 \text { pc, characterize the colors and orbits of } \\
\text { Earths or Super-Earths in the HZ of } 10 \text { nearby } \\
\text { stars. } \\
\text { Colors: measure flux in } 2 \text { bands } \\
\text { with R } 5 \text { and } 20 \% \text { photometric } \\
\text { precision. } \\
\text { Orbits: constrain projected position } \\
\text { to } 30 \text { mas }(0.3 \text { AU at } 10 \text { pc). }\end{array}$ & $\begin{array}{l}\text { At } 3-20 \text { pc, detect }(\mathrm{SNR}>5) \text { EGPs at the } \\
\text { equivalent of } 3 \text { AU. } \\
\text { Search } 100-200 \text { stars for EGPs. } \\
\text { At } 10 \text { pc, measure the colors of EGPs. } \\
\text { Characterize }>5 \text { RV EGPs. } \\
\text { Measure absolute brightness of a } \\
\text { Jupiter with } 10 \% \text { photometric } \\
\text { precision in }>1 \text { band. } \\
\text { Measure relative brightness of a } \\
\text { Jupiter in }>3 \text { bands with R 5 and } \\
5 \% \text { photometric precision. } \\
\text { Detect CH4 in a Jupiter (at } 650 \text { nm } \\
\text { with SNR }=6 \text { ). } \\
\text { Measure continuum flux between } \\
\text { CH4 absorption bands (three } \\
\text { spaced intervals) with SNR }=30 . \\
\text { Detect H2O in a Jupiter at } 830 \text { nm } \\
\text { (20\% deep) with SNR }= \\
\text { (continuum SNR = } 6 \text { ). }\end{array}$ & $\begin{array}{l}\text { At } 1.3 \mathrm{pc} \text {, image disks out to } \sim 2 \mathrm{AU} \text {. } \\
\text { At } 6 \mathrm{pc} \text {, image disks in to } \sim 1 \mathrm{AU} \text {. } \\
\text { Detect spatially resolved } 1 \text { zodi } \\
\text { disks around } 5 \text { nearby stars (detect } \\
\text { } 5 \text { times noise spatially integrated } \\
\text { over field in } \mathrm{R} \sim 5 \text { channel at } 550 \\
\text { nm). } \\
\text { Detect spatially resolved } 5 \text { zodi } \\
\text { disks around } 20 \text { nearby stars (detect } \\
=10 \text { times noise spatially } \\
\text { integrated over field in } \mathrm{R} \sim 5 \\
\text { channel at } 550 \mathrm{~nm} \text { ). } \\
\text { Characterize and constrain optical, physical } \\
\text { and compositional properties. } \\
\text { Take } \mathrm{R} \sim 5 \text { data across the full band. } \\
\text { Polarimetric precision s-P }=0.5^{\circ} \\
\text { and s-q }=5^{\circ} \text { per resolution element } \\
\text { for sources with intrinsic } \\
\text { polarization fractions }>0.01 .\end{array}$ \\
\hline
\end{tabular}

\subsection{Extrasolar Giant Planets}

Detection and characterization of EGPs requires a contrast ratio of $\sim 1 \mathrm{e}-9$, from Equation 1 with $A=0.5, r=7.5 \mathrm{e} 8 \mathrm{~km}$ (5 AU), and $a=7.15 \mathrm{e} 4 \mathrm{~km}(1 \mathrm{RJ})$. PECO's $\sim 20 \lambda / \mathrm{D}$ OWA with a $1.4-\mathrm{m}$ primary aperture allows detection of a $1 \mathrm{RJ}$ gas giant at a separation of $5 \mathrm{AU}$ from a Sun-like star at distances greater than about $3 \mathrm{pc}$. Note that the AU-equivalent separation also depends on stellar luminosity, so a less luminous parent star's 5 AU equivalent may be detectable at a closer distance than $3 \mathrm{pc}$. An EGP with $1 \mathrm{RJ}$ at the equivalent of a 5 AU separation from a Sun-like star should be detectable out to $20 \mathrm{pc}$, with EGPs at greater separations detectable at even further distances. Detection for EGPs is defined as SNR $>5$ for the measurements between 3 and $20 \mathrm{pc}$.

Considering the large range of distances and the fact that integration times are shorter for larger, brighter objects like EGPs compared with terrestrial planets, the number of EGP candidate systems observed may be limited by mission overhead time constraints even with sequential pointing optimization. For example, observations may be limited by the time required to point and thermally and dynamically settle the spacecraft telescope. The EGP detection goal for PECO is to search long enough to achieve SNR $>5$ around 100-200 systems.

Characterization of EGPs in terms of colors requires multiple precise broadband $(\mathrm{R} \sim 5)$ measurements over the spectral range. Absorption features of interest include $\mathrm{CH} 4$ and $\mathrm{H} 2 \mathrm{O}$ [4], with $\mathrm{CH} 4$ bands near 650, 728, 792, and $862 \mathrm{~nm}$ and $\mathrm{H} 2 \mathrm{O}$ at $830 \mathrm{~nm}$. PECO's science goal is to characterize the $650 \mathrm{~nm}$ methane band ( $20 \%$ deep and $\mathrm{R} \sim 20$ wide) and the $830 \mathrm{~nm}$ water band of a Solar System analog Jupiter at $10 \mathrm{pc}$ with $\mathrm{SNR}=6$, which also requires continuum measurements with $\mathrm{SNR}=30$.

\subsection{Debris Disks and Exozodiacal Dust}

Disks of circumstellar material are both precursors and outcomes of the process of planet formation. They have an important role in PECO's characterization of exoplanets and their host systems, as they are both a background signal in exoplanet detection attempts as well as a rich dataset in their own right. Hundreds of Sun-like stars show excess infrared emission due to circumstellar dust and debris disks. Results from Spitzer suggest that many stars have debris disks similar to our own solar system [25]. However, observations of most of these systems remain spatially unresolved and undetected in scattered light [26].

Long wavelength spectral energy distributions are used to derive dust temperatures and covering fractions, but in order to improve the estimated locations of these thermally emissive orbiting dust grains, more information about the dust 
Table 3: Simulation parameters used in this work (cf. [16])

\begin{tabular}{|c|c|c|}
\hline Parameter & Value & Comment \\
\hline Optics throughput & 0.45 & $\begin{array}{l}\text { In addition to, but exclusive of, } \\
\text { coronagraphic throughput (e.g., coatings) }\end{array}$ \\
\hline Imaging wavelength & $\begin{array}{l}450 \text { to } 850 \mathrm{~nm} \\
\mathrm{R}=5\end{array}$ & $\begin{array}{l}\text { Simulations with } 20 \% \text { bandwidth (e.g., } 550 \\
\text { nm with } 110 \mathrm{~nm} \text { ) }\end{array}$ \\
\hline IWA target list cutoff & $2 \lambda / \mathrm{D}$ at $550 \mathrm{~nm}$ & $\begin{array}{l}\text { Cutoff to filter list of candidates prior to } \\
\text { simulation }\end{array}$ \\
\hline Zodiacal background & $22.53 \mathrm{~m}_{\mathrm{V}} / \operatorname{arcsec}^{2}$ & Function of target ecliptic latitude \\
\hline $\begin{array}{l}\text { Exozodiacal dust disk } \\
\qquad \begin{array}{l}1 \text { zodi at HZ } \\
\text { Inner edge } \\
\text { Outer edge } \\
\text { Cloud thickness } \\
\text { Optical depth }\end{array}\end{array}$ & $\begin{array}{l}\text { Scaled in zodi } \\
22.53 \mathrm{~m}_{\mathrm{V}} / \operatorname{arcsec}^{2} \\
0.02 \sqrt{\mathrm{L}} \\
5.0 \sqrt{\mathrm{L}} \\
0.0 \\
\sim r^{-0.34}\end{array}$ & $\begin{array}{l}\text { (Does not apply to specific cases in } 3.6 \text { ) } \\
1 \text { zodi brightness at the HZ, face-on system } \\
\text { (1 zodi used as default case) } \\
\text { L = star bolometric luminosity } \\
\text { Thickness not resolved by telescope } \\
\text { Power law index [34] }\end{array}$ \\
\hline $\begin{array}{l}\text { Radius, semi-major axis, albedo } \\
\text { Earths } \\
\text { Super-Earths } \\
\text { Jupiters }\end{array}$ & $\begin{array}{l}6,400 \mathrm{~km}, 1 \mathrm{AU}, 0.2 \\
12,800 \mathrm{~km}, 1 \mathrm{AU}, 0.2 \\
70,000 \mathrm{~km}, 5 \mathrm{AU}, 0.5\end{array}$ & $\begin{array}{l}\text { Semi-major axis scaled by target system } \sqrt{ } \mathrm{L} \\
\text { Constant albedos }\end{array}$ \\
\hline Planet eccentricity & $e=0^{\circ}$ & No eccentricity \\
\hline Orbit inclination & $i=\pi / 3=60^{\circ}$ & Statistical median for random orientation \\
\hline Detection time and probability & $\begin{array}{l}\text { Time to reach desired } \\
\text { SNR with } 20 \% \\
\text { detection probability } \\
(\mathrm{t} 20)\end{array}$ & $\begin{array}{l}\text { As defined in Section } 3.2 \text { and }[7,16] \text {, planet } \\
\text { detection SNR is computed at each point } \\
\text { along the planet's orbit; the fraction of } \\
\text { points over the orbit that achieve desired } \\
\text { SNR yields detection probability }[32,33]\end{array}$ \\
\hline Detection, characterization & $\mathrm{SNR}>5, \mathrm{SNR}>30$ & As described in Section 2 \\
\hline Wavefront quality & Perfect & No time-variable wavefront errors \\
\hline Detector & Perfect & No readout noise or dark current included \\
\hline
\end{tabular}

morphology and properties of the particles is needed [27]. Spatially resolved scattered light images of disks with polarization information, combined with spectral energy distributions (e.g. from IRAS, Spitzer, and JWST), will constrain the composition, mass, and physical properties of the dust particles [28]. This will in turn yield information on systemic dust mass-loss rates and rates of dust production during epochs of planet formation and dynamical evolution.

The required contrast ratio of $\sim 1 \mathrm{e}-9$ for EGPs supports PECO's debris disk science goal of measuring 1 zodi Solar-System analog disks at distances of at least $\sim 10 \mathrm{pc}$ [7]. Combined with the small IWA, observations with PECO will effectively probe the innermost regions of dust disks to resolve small-scale structures, such as rings, bands, gaps, photocentric offsets, brightness asymmetries, spirality, and non-coplanar features. These structures also provide information about dynamical evolution and the presence of planets, a relationship notably demonstrated around Fomalhaut $[29,30]$ and modeled for the case of terrestrial planets by [31] and others.

The science goal of measuring 1 zodi disks around 5 nearby stars, and 5 zodi disks around 20 nearby stars as close in as a $1 \mathrm{AU}$ equivalent $\mathrm{HZ}$ for systems at a distance of $\sim 6 \mathrm{pc}$ requires an IWA of $\sim 167$ mas. Resolving small-scale inner disk structures is consistent with the terrestrial planet science goals. Disk imaging with an OWA $\sim 20 \lambda / \mathrm{D}$ extends out to $\sim 2$ $\mathrm{AU}$ at $1.3 \mathrm{pc}, \sim 8 \mathrm{AU}$ at $5 \mathrm{pc}$, and $\sim 16 \mathrm{AU}$ at $10 \mathrm{pc}$, and $\sim 32 \mathrm{AU}$ at $20 \mathrm{pc}$. The EGP science goals are consistent with imaging disk structure out to $20 \mathrm{pc}$.

\section{SIMULATED PERFORMANCE}

This section describes several simulations to evaluate the science performance of PECO's medium-class mission design using the high-contrast and small-IWA PIAA coronagraph. For these studies, we have adapted the high-fidelity simulation code described in $[7,16]$ to the PECO mission design and parameters, described in Table 3.

\subsection{Target star list}

The 18,418 candidate target systems used in this work are stars within $100 \mathrm{pc}$ from the Hipparcos catalog. Effective temperatures and bolometric luminosities were used [16] to restrict the list to F, G, K, types and eliminate giants. Candidates listed as sub-arcsecond binaries were also removed; the effect of a close binary companion on observations depends on a number of system specifics that require individual consideration, such as companion magnitude, maximum separation during the scheduled mission with respect to IWA and OWA, etc. The simulation uses the angular size information from the target star list the to calculate the residual scattered starlight for each system.

\subsection{Simulation description}

The PIAA simulations include an inverse PIAA with reimaging optics as described in Section 1.4. The simulation parameters used here are listed in Table 3 , and can be compared with previous work in $[7,16]$. The simulations were 

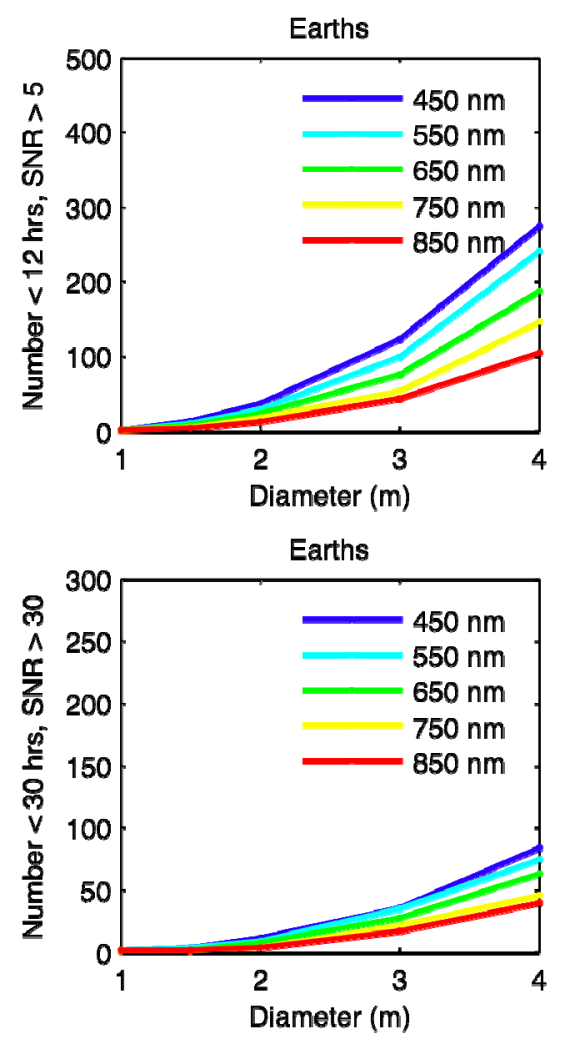

Figure 4: Science performance of a PIAA coronagraph vs. telescope primary D (m) and $\lambda$ for Earths and Super-Earths. Upper panels correspond to Table 4 and lower panels to Table 5. Upper panels show number of Earths (left) and Super-Earths (right) with integration times $<12$ hours to achieve SNR $>5$. Lower panels show number of Earths and Super-Earths with integration times $<30$ hours to achieve SNR $>30$. General simulation parameters are described in Table 3. performed to evaluate trades with wavelength, diameter D, SNR requirements, integration time, planet radius, albedo, and semi-major axis, as well as to evaluate a few specific targets of interest (e.g., Tau Ceti, Alpha Centauri, Epsilon Eridani, disk structures, 55 Cancri).

The simulations take into account residual starlight as a function of target stellar radius, the local zodiacal light as a function of ecliptic latitude, the exozodiacal light (1 zodi equivalent unless noted otherwise), the simulated planet flux, and the coronagraphic throughput. The sampling is of a square grid of points at $0.2 \lambda / \mathrm{D}$ in two orthogonal directions, which gives over 30,000 PSFs in the $20 \lambda / \mathrm{D}$ radius field of view [16]. Binning of the simulation output to PECO's resolution is a post-processing step. Output images from the simulations are consistent with the PECO architecture $(\sim 38$ mas per pixel at $550 \mathrm{~nm}$ ) unless otherwise noted. Table 7 gives mas per pixel as a function of wavelength for $\mathrm{D}=1.4 \mathrm{~m}$ and OWA of $20 \lambda / \mathrm{D}(40 \lambda / \mathrm{D}$ across the image).

The integration time metric we use to evaluate science performance in this work, sometimes referred to as $\mathrm{t} 20$, is a function of planet detection probability and SNR. The SNR is calculated as described in [7, 16], using the photon noise (from the planet, star, zodiacal, and exozodiacal contributions) and the signal (the planet contribution) from all pixels of the detector. The planet detection SNR is obtained by the quadratic sum of the pixel SNRs. The PECO mission design assumes multiple visits of targets for both detection and characterization. The integration time metric used here is therefore not geared toward single-visit completeness. SNR is computed at 100 points along the planet's complete orbit, capturing variation with planetary phase and geometry [16]. The fraction of points that achieve the desired SNR yield the detection probability. The integration time metric used here is the single-exposure integration time required to reach a $20 \%$ probability of detection at the desired SNR (e.g., SNR $=5$ ). With a revisit strategy of 10 visits per target using this integration time, the probability of detection is $90 \%$.

\subsection{Results: Earths and Super-Earths}

The challenges for PECO as a medium-class mission are the science goals of detecting and characterizing terrestrial planets around nearby stars, although they are not the sole focus of the mission. We focus on what kind of terrestrial science performance we can expect from PECO given that the 1.4-m diameter telescope sets the $2 \lambda / \mathrm{D}$ IWA, as described in Table 1. PECO's science goals with respect to terrestrial planets as described in Table 2 are to detect and characterize any planets that may exist around an F, G, K system within the 6 pc neighborhood, and to perform a more broad-based color and orbit classification out to $10 \mathrm{pc}$. 
Table 4: Number of planets with SNR $>5$ and t $20<12$ hrs vs. $\lambda$ and $\mathrm{D}, \mathrm{R}=5$

\begin{tabular}{ccccccccccccc}
\hline $\begin{array}{c}\text { Earths } \\
\text { SNR }>5\end{array}$ & 1.0 & 1.5 & 2.0 & 3.0 & 4.0 & & $\begin{array}{c}\text { Super- } \\
\text { Earths }\end{array}$ & 1.0 & 1.5 & 2.0 & 3.0 & 4.0 \\
\hline $450 \mathrm{~nm}$ & 2 & 14 & 37 & 123 & 275 & & $450 \mathrm{~nm}$ & 6 & 22 & 65 & 202 & 464 \\
$550 \mathrm{~nm}$ & 2 & 11 & 30 & 100 & 242 & & $550 \mathrm{~nm}$ & 4 & 21 & 61 & 191 & 420 \\
$650 \mathrm{~nm}$ & 2 & 8 & 24 & 76 & 188 & & $650 \mathrm{~nm}$ & 3 & 16 & 44 & 160 & 345 \\
$750 \mathrm{~nm}$ & 2 & 5 & 18 & 54 & 147 & & $750 \mathrm{~nm}$ & 2 & 15 & 35 & 121 & 278 \\
$850 \mathrm{~nm}$ & 2 & 4 & 13 & 44 & 106 & & $850 \mathrm{~nm}$ & 2 & 13 & 27 & 87 & 219
\end{tabular}

Table 5: Number of planets with SNR $>30$ and $\mathrm{t} 20<30$ hrs vs. $\lambda$ and $\mathrm{D}, \mathrm{R}=5$

\begin{tabular}{cccccccccccc}
\hline $\begin{array}{c}\text { Earths } \\
\mathrm{SNR}>30\end{array}$ & 1.0 & 1.5 & 2.0 & 3.0 & 4.0 & $\begin{array}{c}\text { Super- } \\
\text { Earths }\end{array}$ & 1.0 & 1.5 & 2.0 & 3.0 & 4.0 \\
\hline $450 \mathrm{~nm}$ & 2 & 4 & 12 & 36 & 84 & $450 \mathrm{~nm}$ & 2 & 13 & 34 & 115 & 264 \\
$550 \mathrm{~nm}$ & 2 & 4 & 9 & 35 & 75 & $550 \mathrm{~nm}$ & 2 & 11 & 30 & 94 & 235 \\
$650 \mathrm{~nm}$ & 2 & 2 & 8 & 28 & 63 & $650 \mathrm{~nm}$ & 2 & 8 & 22 & 71 & 181 \\
$750 \mathrm{~nm}$ & 2 & 2 & 4 & 22 & 46 & $750 \mathrm{~nm}$ & 2 & 5 & 17 & 52 & 146 \\
$850 \mathrm{~nm}$ & 2 & 2 & 4 & 17 & 40 & $850 \mathrm{~nm}$ & 2 & 4 & 13 & 44 & 104
\end{tabular}

It is instructive to consider PECO's capability with a $2 \lambda / \mathrm{D}$ IWA and D $=1.4 \mathrm{~m}$ in context with the performance of the PIAA coronagraph for larger-class missions. Figure 4 shows the number of terrestrial planets (Earths and Super-Earths) that can be detected with a PIAA coronagraph in less than 12 hrs (with SNR > 5) and characterized in less than $30 \mathrm{hrs}$ (with SNR > 30) for diameters up to $\mathrm{D}=4 \mathrm{~m}$. These data are also shown as a function of wavelength, with values in Table 4 and Table 5.

The rationale behind the choice of 12 hours for a detection time is based upon spending a reasonable amount of time observing a system after taking the time to slew, point, acquire, and settle the spacecraft to take the measurement. Similarly, the choice of 30 hours for characterization is based on spending a longer amount of time building up SNR observing a system before having to perform spacecraft station-keeping tasks, such as momentum dumps, adjustments for solar power, or data downlinks. Both higher and lower SNR observations will consist of several smaller blocks of
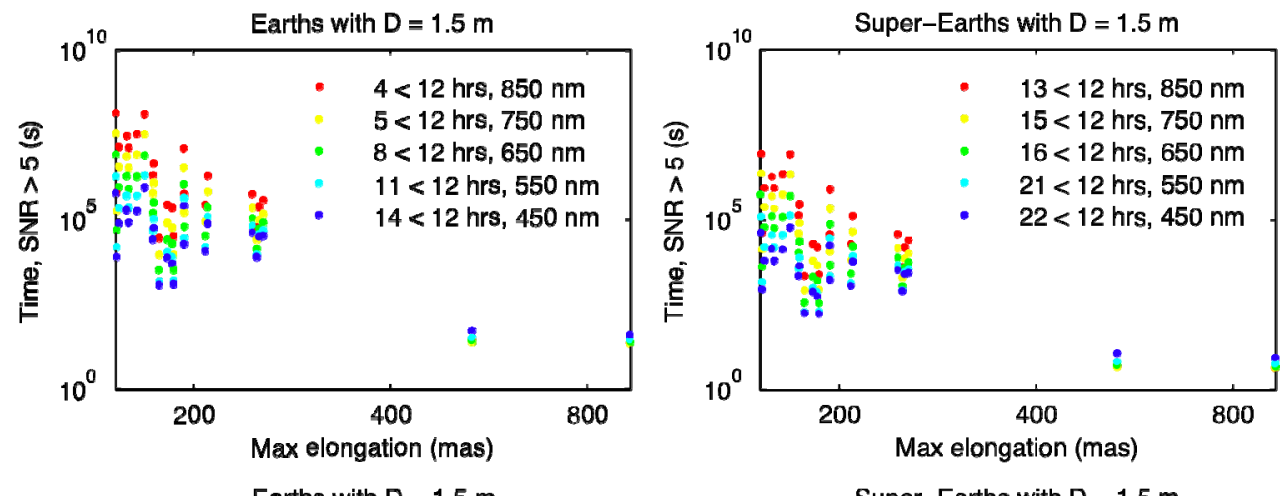
integrations on the order of seconds.
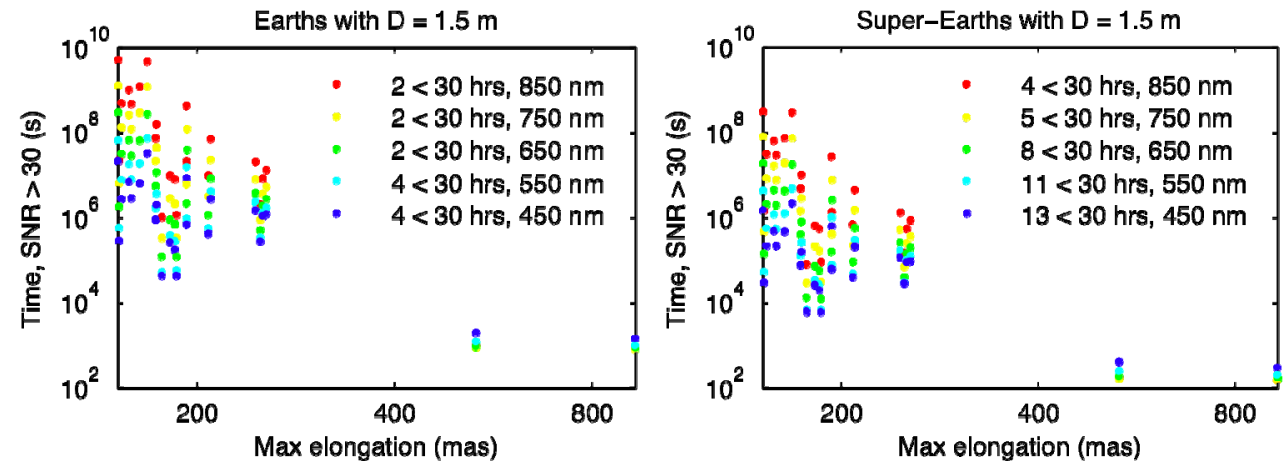

Figure 5: Integration time (t20) vs. max elongation, Earths and Super-Earths, D=1.5 m for SNR > 5 (upper) and SNR > 30 (lower).

Figure 4 shows that the number of targets increases significantly with larger D, as expected. It also shows in detail the dependence on wavelength through PECO's range in the optical. Each simulation was performed for a $20 \%$ bandwidth. Figure 5 shows additional detail of the $\mathrm{D}=1.5 \mathrm{~m}$ case from Figure 4 vs. maximum elongation of the target planet. The four targets requiring the shortest integration times are Alpha Centauri A, Alpha Centauri B, Tau Ceti, and Epsilon Eridani. Additional information on the target systems that satisfy the criteria in these plots can be found in [22] along with detailed lists in [35]. The simulations show that PECO's terrestrial planet science goals are reasonable for a definition of terrestrial planets that includes both Earths and Super-Earths. 

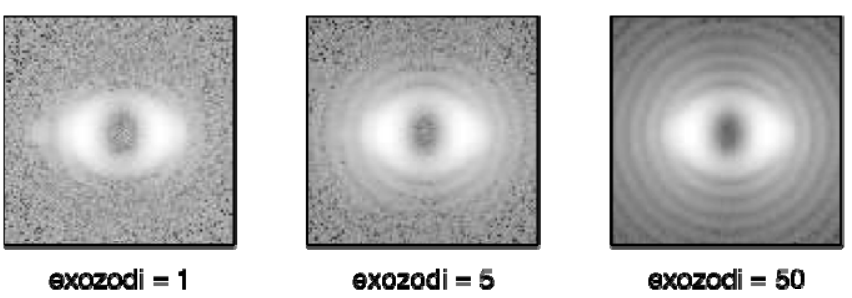

Figure 7: PECO simulated images of 55 Cancri [6] at $12.5 \mathrm{pc}$ with planet $\mathrm{d}$ $\left(\mathrm{Msin} i=3.8 \mathrm{MJ}\right.$ at $5.7 \mathrm{AU}$ ) at $60^{\circ}$ system inclination with exozodiacal dust at levels of 1 zodi, 5 zodi, and 50 zodi after 22 hours integration. Images at $550 \mathrm{~nm} \mathrm{R}=5, \mathrm{IWA}=2 \lambda / \mathrm{D}$ with $\mathrm{D}=1.4 \mathrm{~m}$. Photon noise added, optical throughput 0.45 , scale $\sim 12$ mas/pixel.

\subsection{Results: Radial Velocity}

As shown in Figure 3, there are over a dozen currently known RV planets in the existing target list used for these PECO simulations. There are also a few others that were not already included in the target star list. Table 6 shows results from an oversimplified simulation for the already-included RV target systems as a starting point for evaluation. The simulation uses $1 \mathrm{RJ}$ at $2 \mathrm{AU}$ with 1 zodi of exozodiacal dust at $550 \mathrm{~nm}$ instead of using the estimated

separations and radii of the RV planets. In most cases, the separation and radius assumption we used is conservative from the detection and characterization point of view [22] while the exozodiacal dust assumption is optimistic.

Table 6: Radial velocity systems with $1 \mathrm{RJ}$ at $2 \mathrm{AU}$

\begin{tabular}{|c|c|c|c|c|c|}
\hline \multirow{3}{*}{\multicolumn{2}{|c|}{$\begin{array}{l}\text { Radial Velocity } \\
\text { HIP / Identifier }\end{array}$}} & \multirow[t]{3}{*}{ Dist. (pc) } & $2 \mathrm{AU}$ & t20 (hr) & \multirow{3}{*}{$\begin{array}{l}\text { t20 (hr) } \\
1 \mathrm{RJ} \\
\mathrm{SNR}>30\end{array}$} \\
\hline & & & $550 \mathrm{~nm}$ & & \\
\hline & & & $(\lambda / \mathrm{D})$ & $\mathrm{SNR}>5$ & \\
\hline 1 & 16537 / Eps Eri & 3.2 & 7.7 & 0.00 & 0.08 \\
\hline 2 & 3093 / NSV 245 & 11.1 & 2.2 & 0.11 & 3.78 \\
\hline 3 & 10138 / LHS 13 & 10.9 & 2.3 & 0.13 & 4.52 \\
\hline 4 & 80337 / NSV 7680 & 12.8 & 1.9 & 0.13 & 4.60 \\
\hline 5 & 53721 / 47 Uma & 14.1 & 1.8 & 0.14 & 4.88 \\
\hline 6 & 67275 / Tau Boo A & 15.6 & 1.6 & 0.14 & 4.98 \\
\hline 7 & 43587 / 55 Cancri A & 12.5 & 2.0 & 0.23 & 8.22 \\
\hline 8 & 40693 / LHS 245 & 12.6 & 2.0 & 0.23 & 8.25 \\
\hline 9 & 86796 / LTT 7053 & 15.3 & 1.6 & 0.28 & 10.07 \\
\hline 10 & 109338 / GJ 849 & 15.4 & 1.6 & 0.44 & 15.88 \\
\hline 11 & 98767 / HD 190360 & 15.9 & 1.5 & 0.78 & 28.05 \\
\hline 12 & 27887 / HD 40307 & 12.8 & 1.9 & 0.93 & 33.62 \\
\hline
\end{tabular}

Figure 7 shows the results of a PECO simulation with parameters that correspond to planet $\mathrm{d}$ in the 55 Cancri system (at $12.5 \mathrm{pc}$ ). Planet $\mathrm{d}$ is the largest and most widely separated (Msin $i=3.835 \mathrm{MJ}, 5.77 \mathrm{AU})$ of five detected in the system using the radial velocity method [6]. Exozodiacal dust at levels of 1 zodi (like our Solar System), 5 zodi, and 50 zodi are shown with default system inclination of $60^{\circ}$ in Figure 7 . Images are at $550 \mathrm{~nm}$ for a $1 \mathrm{RJ}$ planet at $5.77 \mathrm{AU}$ with other parameters as in Table 3 . The scale of the images is $\sim 12 \mathrm{mas} /$ pixel. The planet is still visible with 5 zodi for a 22-hour integration, and somewhat less visible with 50 zodi. Figure 6 shows a more detailed comparison of SNR versus exozodiacal level for the

55 Cancri d case. Discussion of the effect of exozodiacal light on exposure time for the general case is treated in [6].

The simulation results shown in Table 6 as well as Figure 6 and Figure 7 support PECO's science goal of detecting and characterizing $>5$ previously-detected RV planets. The simulation results encourage attempts to detect and characterize

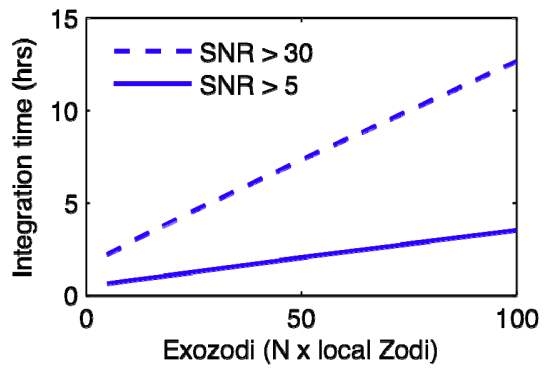

Figure 6: Exozodi vs. integration time for 55 Cancri d [6]. all of the RV planets listed here as well as any others within PECO's IWA range.

\subsection{Results: Jupiters and gas giants}

Simulations performed for Jupiters (1 RJ at 5 AU) yield over 140 systems for which SNR $>5$ is achieved in less than 12 hours (t20) and over 45 systems out to $\sim 20$ pc can be characterized with SNR $>30$ in less than 30 hours [35]. These simulations support PECO's science goals of EGP characterization out to 10 pc. Figure 8 shows simulated images from PECO for three different planet types around Tau Ceti as a function of wavelength. The images show a ten-hour integration with coronagraphic throughput and photon noise (inclusion of the 0.45

optical throughput would proportionally scale the integration time). The image resolution is similar to that expected from PECO.

\subsection{Results: Exozodiacal dust disks}

The ability to detect and/or characterize gas giants at separations of $2 \mathrm{AU}$ and $5 \mathrm{AU}$ (Section 3.4 and Section 3.5) also addresses the nonpolarimetric PECO science goals for exozodiacal dust disks. Figure 9

Table 7: Resolution for simulations

\begin{tabular}{cccc}
$\mathrm{D}=1.4 \mathrm{~m}$ & $\begin{array}{c}20 \lambda / \mathrm{D} \\
(\mathrm{mas})\end{array}$ & $\begin{array}{c}40 \lambda / \mathrm{D} \\
85 \text { pix } \\
\text { (mas/pixel) }\end{array}$ & $\begin{array}{c}40 \lambda / \mathrm{D} \\
265 \text { pix } \\
\text { (mas/pixel) }\end{array}$ \\
\hline $450 \mathrm{~nm}$ & 1326.0 & 31.2 & 10.4 \\
$550 \mathrm{~nm}$ & 1620.7 & 38.1 & 12.7 \\
$650 \mathrm{~nm}$ & 1915.3 & 45.1 & 15.0 \\
$750 \mathrm{~nm}$ & 2210.0 & 52.0 & 17.3 \\
$850 \mathrm{~nm}$ & 2504.6 & 58.9 & 19.6
\end{tabular}



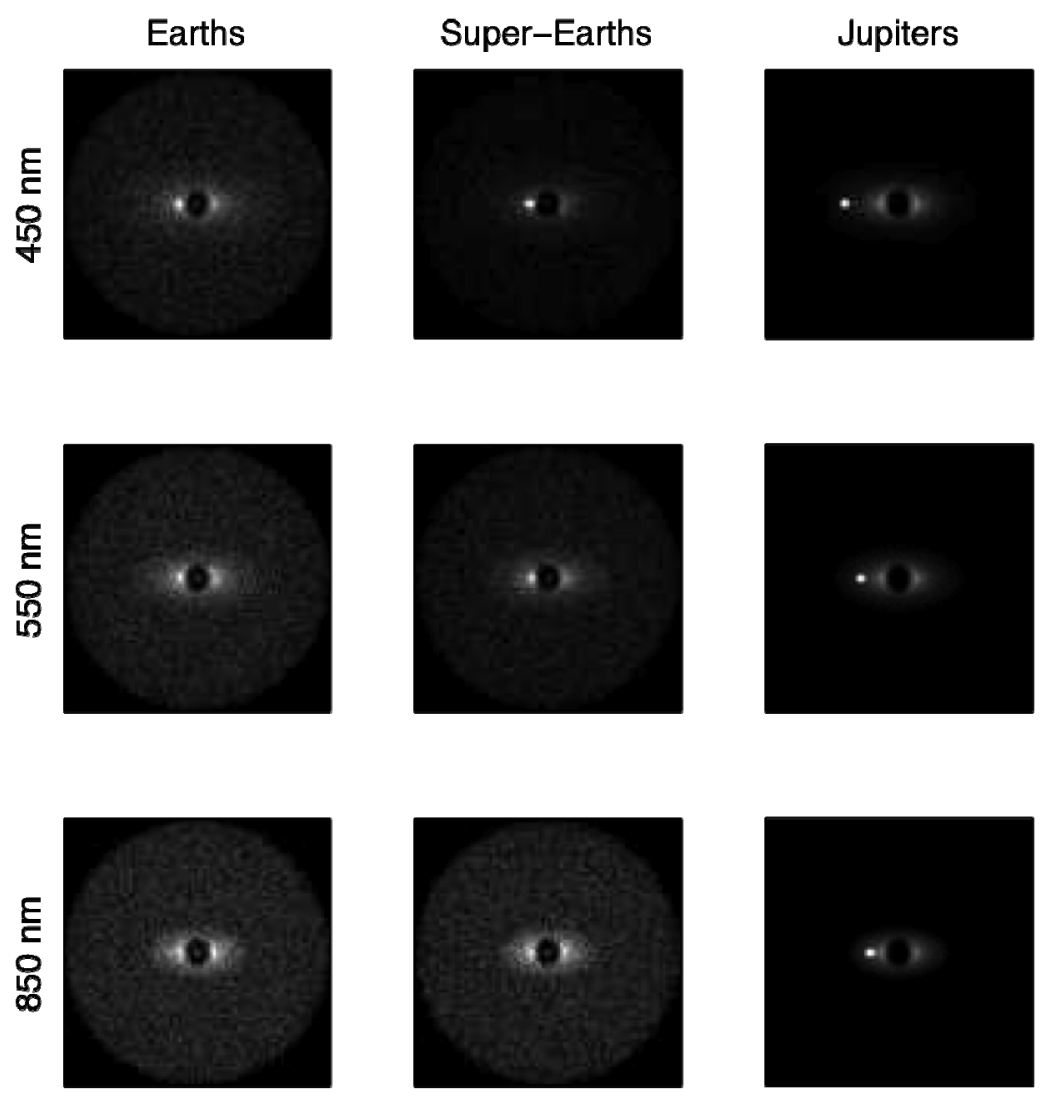

Figure 8: PECO simulations of Earth, Super-Earth, and Jupiter-like planets vs. $\lambda$ at Tau Ceti $(3.65 \mathrm{pc})$ with 1 zodi for local and exozodi, IWA $2 \lambda / \mathrm{D}, 10$-hour integration, coronagraphic throughput and photon noise. Note scale with $\lambda$ in the $85 \times 85$ images: $450 \mathrm{~nm} \sim 31$ mas/pix, $650 \mathrm{~nm} \sim 45 \mathrm{mas} /$ pix, $850 \mathrm{~nm} \sim 59$ mas/pix (see Table 7).

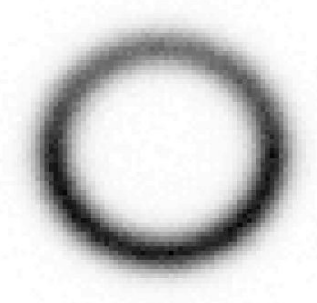

Figure 9: Epsilon Eridani ring simulation with PECO. Ring at 3 AU modeled by G. Schneider. Composition of rocky silicates, width 1 AU, $i=22^{\circ}$ (same as disk), flux density of 1 zodi. Simulated at 550 $\mathrm{nm}, \mathrm{R}=5$, and exposure time of $\sim 3$ hours. shows the result of an example simulation of how PECO might image the inner ring around Epsilon Eridani. Figure 10 shows two simulations with 10 zodi disks, one of a Jupiter and an Earth around the Sun at $10 \mathrm{pc}$ (upper panels) and one of an Earth and a Super-Earth around Alpha Centauri A (lower panels).

\section{SUMMARY}

\subsection{Design Reference Mission}

The science goals, simulation results, three-year nominal mission duration, and the spacecraft orbit and observational constraints contributed to an initial version of a PECO Design Reference Mission (DRM) [35]. Additional details on science rationale, spacecraft and instrument design, and simulation results can be found in $[1,22,35]$.

Table 8 uses the results from the PECO science performance simulation and the science goals to structure the mission into three phases: (1) a Grand Tour of nearby star systems to detect Earths and SuperEarths. (2) a Radial-Velocity follow-up survey, and (3) a Snapshot Survey of giant planet and exozodiacal dust disks. The number of visits per target for the Grand Tour targets is consistent with the use of the t20 integration time metric in the simulations. The number of visits per target for the RV planets is smaller given that there is previously known information about each of the systems. The Snapshot Survey focuses on a larger number of systems to detect and characterize the gas giants and exozodiacal disk structure; the information learned from these observations would greatly aid future searches for planets in these systems. Additional time is allocated for high-SNR characterization of a subset of the Grand Tour targets (using an optimistic 25\% detection rate) and all of the RV targets.

The DRM in Table 8 includes overhead time in addition to integration time with overhead time equal to $50 \%$ of the integration time. The overhead time is based on estimates of time necessary for spacecraft operations: periodic data downlinks to Earth, slewing and thermal/dynamic settling of the spacecraft, target acquisition from star tracker to coarse target acquisition to fine target acquisition and continuous feedback-loop pointing with the LOWFS, and periodic 
Table 8: PECO example design reference mission

\begin{tabular}{|c|c|c|c|c|c|c|c|c|c|}
\hline \multirow{2}{*}{ Mission Phase } & \multicolumn{4}{|c|}{ Initial Detection and Characterization } & \multicolumn{4}{|c|}{ Follow-up High SNR Characterization } & \multirow{2}{*}{$\begin{array}{l}\text { Total } \\
\text { (hours) }\end{array}$} \\
\hline & $\begin{array}{l}\text { Number of } \\
\text { Systems }\end{array}$ & $\begin{array}{l}\text { Integration } \\
\text { (hrs each) }\end{array}$ & $\begin{array}{l}\text { Overhead } \\
\text { (hrs each) }\end{array}$ & $\begin{array}{l}\text { Visits } \\
\text { (each) }\end{array}$ & $\begin{array}{l}\text { Number of } \\
\text { Systems }\end{array}$ & $\begin{array}{l}\text { Integration } \\
\text { (hrs each) }\end{array}$ & $\begin{array}{l}\text { Overhead } \\
\text { (hrs each) }\end{array}$ & $\begin{array}{l}\text { Visits } \\
\text { (each) }\end{array}$ & \\
\hline Commissioning & - & - & - & - & - & - & - & - & 1,440 \\
\hline $\begin{array}{l}\text { Grand Tour Earths } \\
+ \text { Super-Earths }\end{array}$ & 20 & 16 & 8 & 10 & 5 & 400 & 200 & 2 & 10,800 \\
\hline $\begin{array}{l}\text { Follow-up of } \\
\text { Radial Velocity }\end{array}$ & 15 & 16 & 8 & 3 & 15 & 200 & 100 & 2 & 10,080 \\
\hline $\begin{array}{l}\text { Giant Planets }+ \\
\text { Disk Snapshot }\end{array}$ & 120 & 16 & 8 & 1 & - & - & - & - & 2,880 \\
\hline \multicolumn{9}{|c|}{ Total (hours) } & 25,200 \\
\hline & & & & & & & \multirow{2}{*}{\multicolumn{2}{|c|}{$\begin{array}{l}\text { 3-yr mission (hours) } \\
\text { Margin (hours) }\end{array}$}} & 26,280 \\
\hline & & & & & & & & & 1,080 \\
\hline
\end{tabular}

momentum dumps from the reaction wheels. Results of initial Monte Carlo simulations to evaluate scheduling using the spacecraft pointing constraints indicate that the DRM tasks are achievable within the 3-year mission [35].

\subsection{Future work}

Additional studies suggested by this work include simulations of the effects of direct imaging of binary or multiple systems, as well as simulations that analyze performance for all known RV planets with their estimated system parameters. Defining a standard interface between exoplanet data archives and a direct imaging simulation tool would be useful, particularly if it incorporates known information about target circumstellar dust and debris disk distributions. A technology development plan for PECO is also outlined in [1].

\section{ACKNOWLEDGEMENTS}

This research was supported by an appointment to the NASA Postdoctoral Program at the Ames Research Center, administered by ORAU through a contract with NASA and by the Astrophysics Strategic Mission Concept Studies program.
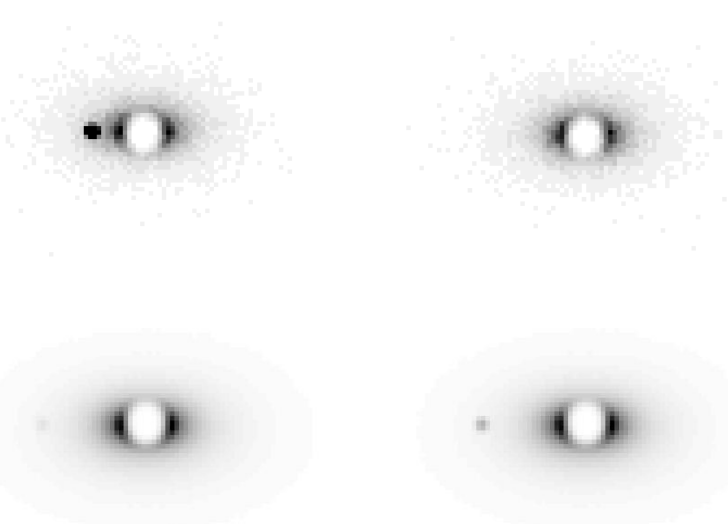

Figure 10: Upper, Jupiter at $5 \mathrm{AU}$ (left) and Earth at 1 AU (right) for Sun at $10 \mathrm{pc}$ with 10 zodi disk, $i=$ $60^{\circ}$ and $24 \mathrm{hr}$ integration. Jupiter far outside IWA where disk is less bright. Earth disappears within the IWA and disk flux. Lower, Earth (left) and Super-Earth (right) both at $1 \mathrm{AU}$ for Alpha Centauri A (G2V) with 10 zodi disk, $i=60^{\circ}, 3 \mathrm{hr}$ exposure.

\section{REFERENCES}

[1] Guyon, O., et al., "PECO Response to the Astro2010 Decadal Survey," http://caao.as.arizona.edu, 1 April 2009.

[2] Woolf, N. et al., "The spectrum of Earthshine: A pale blue dot observed from the ground," Ap. J., 574, 430-433 (2002), doi:10.1086/340929.

[3] Palle, E. et al., "Earth's transmission spectrum from lunar eclipse observations," Nature, 459, 814-816 (2009), doi:10.1038/nature08050.

[4] Karkoschka, E. "Spectrophotometry of the jovian planets and Titan at 300- to 1000-nm wavelength: The methane spectrum," Icarus, 111(1), 174-192 (1994), doi: 10.1006/icar.1994.1139

[5] McKay, C. et al., "The thermal structure of Titan's atmosphere," Icarus 80, $23-53$ (1989), doi: 10.1016/00191035(89)90160-7

[6] Fischer, D. et al., “Five planets orbiting 55 Cancri,” Ap. J., 675(1), 790-801 (2008), doi: 10.1086/525512 
[7] Martinache, F. et al. "Exoplanet imaging with a PIAA Coronagraph. II. Performance,” Ap. J., 639 1129-1137 (2006) doi: $10.1086 / 498408$

[8] Clampin, M. et al., "Hubble Space Telescope ACS Coronagraphic Imaging of the Circumstellar Disk around HD 141569A," Ap. J., 126, 358-392 (2003), doi: 10.1086/375460

[9] Krist, J. et al., "Hubble Space Telescope ACS Coronagraphic Imaging of the AU Microscopii debris disk," The Astronomical Journal, 129, 1008-1017 (2005), doi: 10.1086/426755

[10] Kalas, P. et al., "Optical images of an exosolar planet 25 light-years from Earth," Science 322(5906), 1345-1348 (2008), doi: 10.1126/science.1166609

[11] Krist, J. et al. "Hunting planets and observing disks with the JWST NIRCam Coronagraph," Proceedings of the SPIE, 6693, 66930H-66930H-12 (2007), doi: 10.1117/12.734873

[12] Boffi, F. et al., "ACS Instrument Handbook," version 8.0, Baltimore, STScI (2007)

[13] Sobolev, V., "Light scattering in planetary atmospheres," Oxford and New York, Pergamon Press (International Series of Monographs in Natural Philosophy. Volume 76), 263 p. (1975)

[14] Guyon, O. et al. "Exoplanet imaging with a Phase-induced Amplitude Apodization Coronagraph. I. Principle," Ap. J., 622, 744, (2005), doi: 10.1086/427771

[15] Pluzhnik, E. et al. "Exoplanet imagng with a Phase-induced Amplitude Apodization Coronagraph. III. Diffraction effects and coronagraph design," Ap. J., 644, 1246-1257 (2006), doi: 10.1086/503829

[16] Guyon, O. et al. "Theoretical limits on extrasolar terrestrial planet detection with coronagraphs," Ap. J. Supplement Series, 167(1), 81-99 (2006), doi: 10.1086/507630

[17] Guyon, O. et al. "Coronagraphic low-order wavefront sensor: Principle and application to a phase-induced amplitude coronagraph,” Ap. J., 693, 75-84 (2009), doi: 10.1086/503829

[18] Shaklan, S. and J. Green "Reflectivity and optical surface height requirements in a broadband coronagraph. 1. Contrast floor due to controllable spatial frequencies," App. Opt. 21, 5143-53 (2006), doi: 10.1364/AO.45.005143

[19] Guyon, O., "Phase-induced amplitude apodization of telescope pupils for extrasolar terrestrial planet imaging," Astronomy and Astrophysics, 404, 379-387 (2003), 10.1051/0004-6361:20030457

[20] Vanderbei, R. and W. Traub, "Pupil mapping in two dimensions for high-contrast imaging," Ap. J., 626, 1079-1090 (2005), doi: 10.1086/429909

[21] Shaklan et al., "The Terrestrial Planet Finder Coronagraph dynamics error budget," Proc. SPIE, 5905, 110-121 (2005), doi: 10.1117/12.617890

[22] Guyon, O. et al., "PECO Science Requirements Document," http://caao.as.arizona.edu, 23 April 2009.

[23] Updated 2 July 2009, the Extrasolar PlanetsEncyclopedia, http://exoplanet.eu. Only radial-velocity detected planets with most observational parameters available, including both separation and Msini, were used (315 out of 328).

[24] $\mathrm{Li}, \mathrm{C} .-\mathrm{H}$. et al., "A laser frequency comb that enables radial velocity measurements with a precision of $1 \mathrm{~cm} / \mathrm{s}$," Nature, 452, 610-612 (2008), doi: 10.1038/nature06854

[25] Bryden et al., "Frequency of debris disks around solar-type stars: First results from a Spitzer MIPS survey," Ap. J., 636(2), 1098-1113 (2006), 10.1086/498093.

[26] Meyer, M. et al., "Evolution of mid-IR excess around Sun-like stars: Constraints on models of terrestrial planet formation,” Ap. J., 673(2), L181-L184 (2008), doi: 10.1086/527470

[27] Backman, D. and Paresce, F., "Main sequence stars with circumstellar solid material: the VEGA phenomenon," Protostars and planets III (A93-42937 17-90), 1253-1304 (1993).

[28] Schneider, G. et al., "Discovery of an 86 AU radius debris ring around HD 181327," Ap. J., 650(1), 414-431 (2006), doi: $10.1086 / 506507$

[29] Kalas, P. et al., "A planetary system as the origin of structure in Fomalhaut's dust belt," Nature, 435, 1067-1070 (2005), doi:10.1038/nature03601

[30] Kalas, P. et al., "Optical images of an exosolar planet 25 light-years from Earth," Science, 322(5906), 1345- (2008), doi: 10.1126/science.1166609

[31] Kuchner, M. and M. Holman, “The geometry of resonant signatures in debris disks with planets," Ap. J., 588(2), 1110-1120 (2003), doi: 10.1086/374213

[32] Brown, R., “Obscurational completeness,” Ap. J., 607(2), 1003-1013, (2004), 1013, doi: 10.1086/383586

[33] Brown, R., "Single-visit photometric and obscurational completeness," Ap. J., 624(2), 1010-1024 (2005), doi: 10.1086/429124]

[34] Kelsall, T. et al., "The COBE diffuse infrared background experiment search for the cosmic infrared background, II. Model of the interplanetary dust cloud," Ap. J., 508(1), $44-73$ (1998), doi: 10.1086/306380

[35] Cahoy, K. et al., "PECO Design Reference Mission," http://caao.as.arizona.edu, 23 March 2009. 